\title{
Estado nutricional antropométrico e associação com pressão arterial em crianças e adolescentes: um estudo populacional
}

\author{
Anthropometric nutritional status and association with blood pressure \\ in children and adolescents: a population-based study
}

\author{
Letícia da Silva Souza ${ }^{1}$, Rafaela Cavalheiro do Espírito Santo ${ }^{\natural} \bowtie$, Camila Franceschi ${ }^{3}$, Cassiane de Avila ${ }^{4}$, \\ Suellienn Centenaro ${ }^{5}$, Gisselle Soares dos Santos ${ }^{6}$ \\ 1 Departamento de Nutrição da Universidade Federal do Rio Grande do Sul (UFRGS). Porto Alegre, RS. \\ 2 Programa de Pós Graduação em Medicina: Ciências Médicas da UFRGS. Porto Alegre, RS. \\ ${ }^{3}$ Departamento de Alimentação Coletiva da Cooperativa Mista de Produção, Industrialização e Comercialização de Biocombustíveis do Brasil (COOPERBIO). Seberi, RS. \\ ${ }^{4}$ Departamento de Ciências da Saúde da Universidade Regional Integrada do Alto Uruguai e das Missões (URI). Palmitinho, RS \\ ${ }^{5}$ Departamento de Nutrição do Hospital Regional São Paulo da ASSEC. Xanxerê, SC. \\ Curso de Nutrição da Universidade Federal de Santa Maria (UFSM). Santa Maria, RS.
}

\section{RESUMO}

Objetivos: Avaliar o estado nutricional de crianças e de adolescentes por meio de indicadores antropométricos e avaliar a associação desses resultados com a pressão arterial.

Métodos: Foram incluídos neste estudo crianças e adolescentes entre quatro e 19 anos de idade, oriundos de escolas rurais e urbanas de um município do Rio Grande do Sul. Foram excluídos aqueles que recusaram (ou cujos pais recusaram) participar ou não assinaram o termo de consentimento livre e esclarecido, ou nos quais não foi possível realizar as medidas antropométricas ou da pressão arterial. Foram mensurados peso, estatura, circunferência da cintura, pregas cutâneas e pressão arterial, e foi calculado o índice de massa corporal. Os dados foram descritos por média, desvio padrão, amplitude de variação, frequências absolutas e relativas. Foi realizada estratificação por sexo, índice de massa corporal e obesidade central, para analisar a influência destas variáveis sobre as demais. O Qui-quadrado de Pearson foi utilizado para avaliar as associações. O nível de significância foi de $\mathrm{p}<0,05$.

Resultados: No período da coleta de dados estavam matriculados nas escolas do município 7.082 crianças e adolescentes dentro da faixa etária determinada, os quais foram convidados a participar. Após aplicação dos critérios de exclusão, 5.936 crianças/adolescentes foram incluídos no estudo. A amostra apresentou prevalência de excesso de peso de 30,3\%, obesidade central $31,1 \%$ e excesso de gordura corporal $8,7 \%$. $\mathrm{O}$ excesso de peso foi maior em crianças de escolas particulares $(44,1 \%)$ quando comparadas com escolas estaduais $(29,5 \%)$ e municipais $(29,9 \%)(p<0,001)$. Foram encontradas associações estatisticamente significativas de hipertensão arterial com sobrepeso, obesidade e obesidade central $(\mathrm{p}<0,001)$.

Conclusões: A prevalência de excesso de peso foi elevada e a hipertensão arterial associou-se com sobrepeso, obesidade e obesidade central nas crianças e adolescentes da população estudada.

DESCRITORES: estado nutricional; criança; adolescente; obesidade; hipertensão; pressão arterial.

\section{ABSTRACT}

Aims: To evaluate the nutritional status of children and adolescents by anthropometric indicators and to evaluate the association of these results with blood pressure.

Methods: Children and adolescents between four and 19 years of age from rural and urban schools of a municipality of Rio Grande do Sul were included in this study. Those who refused (or whose parents refused) to participate or did not sign the consent form, or in whom it was not possible to carry out the anthropometric or blood pressure measurements, were excluded. Weight, height, waist circumference, skin folds and blood pressure were measured, and the body mass index was calculated. Data were described by mean, standard deviation, amplitude of variation, absolute and relative frequencies. Stratification by gender, body mass index and central obesity was performed to analyze the influence of these on the other variables. Pearson's Chi-square was used to evaluate the associations. The level of significance was $p<0.05$.

Results: At the time of data collection, 7,082 children and adolescents within the age group were enrolled in the municipal schools, and they were invited to participate. After applying the exclusion criteria, 5,936 children/adolescents were included in the study. The sample presented prevalence of overweight of $30.3 \%$, central obesity $31.1 \%$ and excess body fat $8.7 \%$. Overweight was higher in children from private schools $(44.1 \%)$ than in state schools $(29.5 \%)$ and municipal schools $(29.9 \%)(\mathrm{p}<0.001)$. There were statistically significant associations of high blood pressure with overweight, obesity, and central obesity $(\mathrm{p}<0.001)$.

Conclusions: Prevalence of overweight was high and hypertension was associated with overweight, obesity and central obesity in children and adolescents of the studied population.

KEY WORDS: nutritional status; child; adolescent; obesity; hypertension; blood pressure, high.

Recebido: outubro, 2016

Aceito: março, 2017 
Abreviaturas: IMC, índice de massa corporal; OMS, Organização Mundial da Saúde; PA, pressão arterial; PAS, pressão arterial sistólica; PAD, pressão arterial diastólica; \%GC, percentual de gordura corporal.

\section{INTRODUÇÃO}

O acúmulo excessivo ou anormal de gordura corporal, classificado como sobrepeso, obesidade ou obesidade central, pode acarretar risco para a saúde. Considerado um problema de saúde pública, tem origem da interação de fatores genéticos, metabólicos, ambientais e comportamentais, e psicológicos $[1,2]$.

Nas últimas décadas, devido ao avanço das tecnologias e as transições demográficas e epidemiológicas, a transição nutricional vivenciada pelo Brasil reflete um aspecto de insegurança alimentar e nutricional não só na população adulta, mas também em crianças e adolescentes $[3,4]$. A transição nutricional é um fenômeno no qual ocorre uma inversão nos padrões de distribuição dos problemas nutricionais de uma dada população no tempo, ou seja, uma mudança na magnitude e no risco atribuível de agravos associados ao padrão de determinação de doenças atribuídas ao atraso e à modernidade, sendo em geral, uma passagem da desnutrição para a obesidade [5].

A obesidade infantil é fator de risco para obesidade na idade adulta e, segundo Rivera et al. [6], em estudo publicado em 2014, entre 42,4 e 51,8 milhões de crianças e adolescentes (0-19 anos) na América Latina e Caribe estavam acima do peso recomendado para idade, representando 20,0 a $25,0 \%$ da população total de crianças e de adolescentes da região. No Brasil, a prevalência de excesso de peso varia de $8,9 \%$ a $31,3 \%$ em crianças e em adolescentes [7-9]. Além disto, estima-se que $11 \%$ das crianças menores de cinco anos de todo o mundo terão sobrepeso no ano de 2025 [10].

Diante deste quadro, faz-se importante a detecção precoce de alterações do estado nutricional na infância, com intuito de estabelecer estratégias de prevenção e tratamento, não só do excesso de peso como das doenças associadas ao mesmo. O presente estudo teve como objetivo avaliar o estado nutricional antropométrico de crianças e de adolescentes de um município do Rio Grande do Sul e avaliar a associação desses indicadores com os níveis de pressão arterial (PA).

\section{MÉTODOS}

Este estudo populacional transversal foi conduzido em Palmeira das Missões, Rio Grande do Sul, município com população de 34.328 habitantes, área de $1.419,430 \mathrm{~km}^{2}$ e densidade demográfica de 24,18 habitantes $/ \mathrm{km}^{2}$ em 2010. A população em estudo foi constituída por crianças e adolescentes oriundas de sete escolas rurais e 19 escolas urbanas (12 escolas estaduais, 10 municipais e quatro particulares). A coleta de dados ocorreu entre outubro de 2010 e junho de 2012 e abrangeu todas as escolas do município, inclusive as rurais com difícil acesso. O estudo foi aprovado pelo Comitê de Ética e Pesquisa da Universidade Federal de Santa Maria, pelo parecer consubstanciado CEP-UFSM: 23081.004617/2010-27, e todos os participantes ou seus responsáveis assinaram um termo de consentimento livre e esclarecido.

Como critério de inclusão, adotou-se a faixa etária entre quatro anos completos e 19 anos e 11 meses. Os critérios de exclusão do estudo foram: recusa em participar, ou não assinatura do termo de consentimento; impossibilidade de realizar as medidas antropométricas (indivíduos com tala de gesso, cadeirantes); e dificuldade na avaliação da PA (alunos que recém tinham participado de aula de educação física). As crianças e adolescentes incluídos no estudo foram estratificados por idade segundo os critérios do Ministério da Saúde, que considera, como pré-escolares, crianças de dois anos a cinco anos e 11 meses; escolares, crianças de seis anos a 10 anos e 11 meses; e adolescentes jovens, de 11 anos a 19 anos e 11 meses [9].

Todas as medidas antropométricas e a avaliação da PA foram realizadas por um grupo de alunos do Curso de Nutrição da Universidade Federal de Santa Maria, Campus de Palmeira das Missões, Rio Grande do Sul, previamente capacitados segundo as normas da Organização Mundial da Saúde (OMS), sob orientação e supervisão da professora orientadora. Os dados antropométricos avaliados foram peso, estatura, índice de massa corporal (IMC), circunferência da cintura para avaliar obesidade central e prega cutânea para avaliar percentual de gordura corporal (\%GC).

O peso corporal foi aferido em balança portátil com graduação em 10 gramas (G-Tech, Rio de Janeiro), com os participantes descalços, vestindo roupas leves. A estatura foi mensurada com estadiômetro portátil (Sanny, São Paulo), com os participantes descalços e com os pés juntos, os calcanhares contra a parede. Os valores lidos foram arredondados para o $0,5 \mathrm{~cm}$ mais próximo. O IMC foi calculado pela fórmula peso/estatura ${ }^{2}$ e os resultados foram aplicados na curva de IMC/idade da OMS. Para a classificação foi utilizado o escore $\mathrm{Z}$ considerando as curvas de referência da OMS 2006 [12]. A classificação do IMC pelo escore $\mathrm{Z}$ de acordo com as curvas da OMS é a seguinte: 
normal $=2<$ escore $Z>-2$; magreza $=-3 \leq$ escore $Z$ $\leq-2$; magreza severa $=$ escore $Z<-3$; sobrepeso $=1$ $<$ escore $Z \leq 2$; obesidade $=$ escore $Z>2$.

A circunferência da cintura foi medida com fita métrica não elástica no ponto médio entre a última costela e a crista ilíaca, sendo realizada a leitura no final da expiração com o indivíduo respirando normalmente. Para indivíduos de 4 a 16 anos, a classificação foi em percentis segundo McCarthy et al. [13]. Para indivíduos acima dos 16 anos, foi utilizada a classificação com pontos de corte de $\geq 90 \mathrm{~cm}$ para homens e $\geq 80 \mathrm{~cm}$ para mulheres [14]. Um valor maior ou igual ao percentil 90 indicou a presença de obesidade central.

Para a aferição das pregas cutâneas foi utilizado um plicômetro científico (Cescorf, Porto Alegre, RS), com alta precisão e sensibilidade de $0,1 \mathrm{~mm}$. Cada prega cutânea foi aferidas três vezes consecutivas e foi utilizada a média entre as três aferições. A prega cutânea tricipital foi medida na face posterior do braço não dominante, na metade da linha que liga o processo acromial ao olécrano e a prega cutânea subescapular foi medida logo abaixo do ângulo inferior da escápula. A pele foi levantada $1 \mathrm{~cm}$ abaixo do ângulo inferior da escápula, de tal forma que se pudesse observar um ângulo de $45^{\circ}$ entre esta e a coluna vertebral. O \%GC foi estimado através da utilização das fórmulas propostas por Slaughter et al. [15]. A classificação do \%GC foi realizada de acordo com a proposta por Lohman et al. [16].

A pressão arterial sistólica (PAS) e a pressão arterial diastólica (PAD) foram medidas pelo método auscultatório e classificadas conforme as recomendações das VI Diretrizes Brasileiras de Hipertensão Arterial $[17,18]$. A PA foi aferida preferencialmente com a criança tranquila, após cinco a 10 minutos de repouso, na posição sentada e com o braço direito estendido na altura do coração. Foram realizadas duas ou mais aferições no mesmo participante em ocasiões diferentes, e a média foi utilizada nas análises estatísticas. Foram considerados hipertensos os indivíduos que apresentaram pressão arterial acima do percentil 95 de acordo com a idade, o sexo e a estatura [18].

As variáveis quantitativas foram descritas por média, desvio padrão (DP) e amplitude de variação. As variáveis categóricas foram descritas por frequências absolutas e relativas. Foi realizada estratificação por sexo, IMC e obesidade central para analisar a influência destas variáveis sobre as demais. O Qui-quadrado de Pearson foi usado para avaliar a associação entre as variáveis. Foi aplicado teste dos resíduos ajustados em caso de significância estatística. O nível de significância adotado foi de $5 \%(p<0,05)$ e as análises foram realizadas no programa IBM SPSS Statistics versão 21.0 .

\section{RESULTADOS}

No período da coleta de dados estavam matriculados nas escolas do município 7.082 crianças e adolescentes dentro da faixa etária determinada para inclusão no estudo, os quais foram convidados a participar. Desta amostra, 1.145 alunos ou seus responsáveis não aceitaram participar e/ou não assinaram o termo de consentimento e em um estudante não foi possível aferir a PA. Sendo assim, foram incluídos 5.936 participantes, com média de idade de $11,3 \pm 3,6$ anos, sendo $50,7 \%$ do sexo feminino. Os participantes foram estratificados em pré-escolares (433 crianças; 7,3\%), escolares (1.841 crianças; $31,0 \%$ ) e adolescentes (3.662 crianças; $61,7 \%$ ).

Entre os 5.936 participantes, $4.354(73,3 \%)$ eram oriundos de escolas estaduais, $1.294(21,8 \%)$ de escolas municipais e $288(4,9 \%)$ de escolas particulares. As proporções de excesso de peso, obesidade central e \%GC estão descritas na Tabela 1.

Tabela 1. Caracterização antropométrica de 5.936 crianças e adolescentes com idade entre quatro de 19 anos oriundas de escolas rurais e urbanas do município de Palmeira das Missões, RS, avaliadas entre outubro/2010 e junho/2012.

\begin{tabular}{lc}
\hline \multicolumn{1}{c}{ Variáveis } & Resultados \\
\hline Estatura $(m)$ - média \pm DP [mínimo-máximo] & $1,44 \pm 0,19[0,95-1,89]$ \\
Classificação estatura (z-score) - n (\%) & \\
Baixa estatura grave & $24(0,4)$ \\
Baixa estatura moderada & $206(3,5)$ \\
Eutrofia & $5.542(93,4)$ \\
Estatura acima da média da população & $164(2,8)$ \\
IMC (kg/m²) & $19,4 \pm 4,0[9,5-57,5]$ \\
Classificação IMC (z-score) - n (\%) & $12(0,2)$ \\
Magreza Severa & $102(1,7)$ \\
Magreza & $4.023(67,8)$ \\
Eutrofia & $1.124(18,9)$ \\
Sobrepeso & $675(11,4)$ \\
Obesidade & $65,0 \pm 9,8[25-120]$ \\
Cintura (cm) - média $\pm D P$ [mínimo-máximo] & \\
Classificação circunferência da cintura & $1.845(31,1)$ \\
Com obesidade central & $4.091(68,9)$ \\
Sem obesidade central & $17,8 \pm 7,1[3,2-110]$ \\
\%GC- média $\pm D P$ [mínimo-máximo] & \\
Classificação \%GC - n (\%) & $247(4,2)$ \\
Muito baixo & $961(16,2)$ \\
Baixo & $3.609(60,8)$ \\
Ótimo/adequado & $601(10,1)$ \\
Moderadamente alto & $331(5,6)$ \\
Alto/excesso & $186(3,1)$ \\
Muito alto/obeso & \\
\hline
\end{tabular}

DP, desvio padrão; IMC, índice de massa corporal; \%GC, percentual de gordura corporal. 
Foi encontrada associação estatisticamente significativa entre o tipo de escola e a classificação do IMC. Sobrepeso e obesidade foram mais prevalentes em oriundos de escolas particulares (sobrepeso $25,3 \%$; obesidade 18,8\%) quando comparados aos de escolas estaduais (sobrepeso 18,8\%; obesidade 10,7\%) e de escolas municipais (sobrepeso 18,1\%; obesidade 11,8\%) $(\mathrm{p}<0,001)$. O nível de magreza foi maior em oriundos de escolas estaduais $(2,1 \%)$ quando comparados com os de escolas particulares $(1,7 \%)$ e de escolas municipais $(1,2 \%)(p<0,05)$.

Em relação ao sexo, observou-se que meninas apresentaram maior proporção de sobrepeso e obesidade central, entretanto baixo e muito baixo \%GC. Os meninos apresentaram mais frequentemente obesidade e \%GC alto e muito alto, além de hipertensão arterial estágio II (Tabela 2).

Houve associação entre idade pré-escolar e sobrepeso, estatura acima da média da população, obesidade central, percentual de gordura corporal moderadamente alto e alto e hipertensão arterial nos dois estágios. Entre os indivíduos com obesidade, a maior proporção foi de crianças em idade pré-escolar e escolar, estatura acima da média da população, obesidade central, \%GC moderadamente alto ou maior e hipertensão arterial nos dois estágios (Tabela 3).

A maioria dos indivíduos com obesidade central estava em idade escolar e se associaram a estatura acima da média da população, \%GC moderadamente alto ou maior e hipertensão arterial em qualquer um dos dois estágios (Tabela 4).

Considerando as variáveis obesidade, obesidade central, alto \%GC e hipertensão arterial como fatores de risco para doenças metabólicas em geral, observou-se que 188 crianças $(3,2 \%)$ as possuíam concomitantemente.

Tabela 2. Associação da variável sexo com as variáveis estatura, índice de massa corporal, circunferência da cintura, porcentagem de gordura corporal e pressão arterial de 5.936 crianças e adolescentes com idade entre quatro de 19 anos, oriundas de escolas rurais e urbanas do município de Palmeira das Missões, RS, avaliadas entre outubro de 2010 e junho de 2012.

\begin{tabular}{|c|c|c|c|}
\hline Variáveis & $\begin{array}{l}\text { Sexo masculino } \\
(\mathrm{n}=2.926) \\
\mathrm{n}(\%)\end{array}$ & $\begin{array}{c}\text { Sexo feminino } \\
(\mathrm{n}=3.010) \\
\mathrm{n}(\%)\end{array}$ & $\mathbf{p}^{\#}$ \\
\hline Classificação da estatura (z-score) & & & 0,235 \\
\hline Baixa estatura grave & $9(0,3)$ & $15(0,5)$ & \\
\hline Baixa estatura moderada & $97(3,3)$ & $109(3,6)$ & \\
\hline Eutrofia & $2.729(93,3)$ & $2.813(93,5)$ & \\
\hline Estatura acima da média da população & $91(3,1)$ & $73(2,4)$ & \\
\hline Classificação do IMC (z-score) & & & $<0,001$ \\
\hline Magreza Severa & $8(0,3)$ & $4(0,1)$ & \\
\hline Magreza & $55(1,9)$ & $47(1,6)$ & \\
\hline Eutrofia & $1.962(67,1)$ & $2.061(68,5)$ & \\
\hline Excesso de peso & $901(30,8)$ & $898(29,8)$ & \\
\hline Classificação da circunferência da cintura & & & $<0,001$ \\
\hline Com obesidade central & $846(28,9)$ & $999(33,2)$ & \\
\hline Sem obesidade central & $2.080(71,1)$ & $2.011(66,8)$ & \\
\hline Classificação do \%GC & & & $<0,001$ \\
\hline Muito baixa & $11(0,4)$ & $236(7,8)^{*}$ & \\
\hline Baixa & $431(14,7)$ & $530(17,6)^{*}$ & \\
\hline Ótimo/adequada & $1.896(64,8)^{*}$ & $1.713(56,9)$ & \\
\hline Moderadamente alta & $274(9,4)$ & $327(10,9)$ & \\
\hline Alta/excesso & $196(6,7)^{*}$ & $135(4,5)$ & \\
\hline Muito alta/obeso & $118(4,0)^{*}$ & $68(2,3)$ & \\
\hline Classificação da pressão arterial & & & $<0,001$ \\
\hline Ótima & $12(0,4)$ & $12(0,4)$ & \\
\hline Normal & $1.495(51,1)$ & $1.691(56,2)^{*}$ & \\
\hline Limítrofe & $628(21,5)$ & $648(21,5)$ & \\
\hline Hipertensão estágio I & $247(8,4)$ & $233(7,7)$ & \\
\hline Hipertensão estágio II & $544(18,6)^{*}$ & $426(14,2)$ & \\
\hline
\end{tabular}

IMC, índice de massa corporal; \%GC, percentual de gordura corporal.

\# Teste qui-quadrado de Pearson. * Associação significativa pelo teste dos resíduos ajustados a 5\% de significância. 
Tabela 3. Associação da variável índice de massa corporal com as variáveis faixa etária, estatura, circunferência da cintura, porcentual de gordura corporal e pressão arterial de 5.936 crianças e adolescentes com idade entre quatro de 19 anos, oriundas de escolas rurais e urbanas do município de Palmeira das Missões, RS, avaliadas entre outubro de 2010 e junho de 2012.

\begin{tabular}{|c|c|c|c|c|c|}
\hline Variáveis & $\begin{array}{c}\text { Magreza } \\
(\mathrm{n}=114) \\
\mathrm{n}(\%)\end{array}$ & $\begin{array}{c}\text { Eutrofia } \\
(n=4.023) \\
n(\%)\end{array}$ & $\begin{array}{c}\text { Sobrepeso } \\
\begin{array}{c}(n=1.124) \\
n(\%)\end{array}\end{array}$ & $\begin{array}{c}\text { Obesidade } \\
(n=675) \\
n(\%)\end{array}$ & $\mathbf{p}^{\#}$ \\
\hline Faixa etária & & & & & $<0,001$ \\
\hline Pré-escolar & $4(3,5)$ & $255(6,3)$ & $109(9,7)^{*}$ & $65(9,6)^{*}$ & \\
\hline Escolar & $34(29,8)$ & $1219(30,3)$ & $342(30,4)$ & $246(36,4)^{*}$ & \\
\hline Adolescente & $76(66,7)$ & $2549(63,4)^{*}$ & $673(59,9)$ & $364(53,9)$ & \\
\hline Classificação da estatura (z-score) & & & & & $<0,001$ \\
\hline Baixa estatura grave/moderada & $10(8,8)^{*}$ & $186(4,6)^{*}$ & $26(2,3)$ & $8(1,2)$ & \\
\hline Estatura na média da população & $103(90,4)$ & $3.776(93,9)^{*}$ & $1.056(94,0)$ & $607(89,9)$ & \\
\hline Estatura acima da média da população & $1(0,9)$ & $61(1,5)$ & $42(3,7)^{*}$ & $60(8,9)^{*}$ & \\
\hline Classificação da circunferência da cintura & & & & & $<0,001$ \\
\hline Com obesidade central & $5(4,4)$ & $400(9,9)$ & $804(71,5)^{*}$ & $636(94,2)^{*}$ & \\
\hline Sem obesidade central & $109(95,6)^{*}$ & $3.623(90,1)^{*}$ & $320(28,5)$ & $39(5,8)$ & \\
\hline Classificação do \%GC & & & & & $<0,001$ \\
\hline Muito baixo & $26(22,8)^{*}$ & $212(5,3)^{*}$ & $8(0,7)$ & $1(0,1)$ & \\
\hline Baixo & $55(48,2)^{*}$ & $863(21,5)^{*}$ & $39(3,5)$ & $4(0,6)$ & \\
\hline Ótimo/adequado & $32(28,1)$ & $2.772(68,9)^{*}$ & $662(58,9)$ & $143(21,2)$ & \\
\hline Moderadamente alto & $0(0,0)$ & $149(3,7)$ & $288(25,6)^{*}$ & $164(24,3)^{*}$ & \\
\hline Alto/excesso & $1(0,9)$ & $26(0,6)$ & $107(9,5)^{*}$ & $197(29,2)^{*}$ & \\
\hline Muito alto/obeso & $0(0,0)$ & $0(0,0)$ & $20(1,8)$ & $166(24,6)^{*}$ & \\
\hline Classificação da pressão arterial & & & & & $<0,001$ \\
\hline Ótima & $0(0,0)$ & $21(0,5)^{*}$ & $1(0,1)$ & $2(0,3)$ & \\
\hline Normal & $79(69,3)^{*}$ & $2.414(60,0)^{*}$ & $504(44,8)$ & $189(28,0)$ & \\
\hline Limítrofe & $18(15,8)$ & $854(21,2)$ & $256(22,8)$ & $148(21,9)$ & \\
\hline Hipertensão estágio I & $7(6,1)$ & $275(6,8)$ & $124(11,0)^{*}$ & $74(11,0)^{*}$ & \\
\hline Hipertensão estágio II & $10(8,8)$ & $459(11,4)$ & $239(21,3)^{*}$ & $262(38,8)^{*}$ & \\
\hline
\end{tabular}

\%GC, percentual de gordura corporal. \# Teste qui-quadrado de Pearson. * Associação significativa pelo teste dos resíduos ajustados a 5\% de significância.

Tabela 4. Associação da variável obesidade central com as variáveis faixa etária, estatura, percentual de gordura corporal e pressão arterial de 5.936 crianças e adolescentes com idade entre quatro de 19 anos, oriundas de escolas rurais e urbanas do município de Palmeira das Missões, RS, avaliadas entre outubro de 2010 e junho de 2012.

\begin{tabular}{|c|c|c|c|}
\hline Variáveis & $\begin{array}{c}\text { Com obesidade central } \\
(\mathbf{n}=1845) \\
\mathbf{n}(\%)\end{array}$ & $\begin{array}{c}\text { Sem obesidade central } \\
(\mathrm{n}=4.091) \\
\mathrm{n}(\%)\end{array}$ & $\mathbf{p}^{\#}$ \\
\hline Faixa etária & & & 0,001 \\
\hline Pré-escolar & $115(6,2)$ & $318(7,8)^{*}$ & \\
\hline Escolar & $630(34,1)^{*}$ & $1.211(29,6)$ & \\
\hline Adolescente & $1.100(59,6)$ & $2.562(62,6)^{*}$ & \\
\hline Classificação estatura (z-score) & & & $<0,001$ \\
\hline Baixa estatura grave/moderada & $16(0,9)$ & $214(5,2)^{*}$ & \\
\hline Estatura na média da população & $1.710(92,7)$ & $3.832(93,7)$ & \\
\hline Estatura acima da média da população & $119(6,4)^{*}$ & $45(1,1)$ & \\
\hline Classificação do \%GC & & & $<0,001$ \\
\hline Muito baixo & $9(0,5)$ & $238(5,8)^{*}$ & \\
\hline Baixo & $46(2,5)$ & $915(22,4)^{*}$ & \\
\hline Ótimo/adequado & $858(46,5)$ & $2751(67,3)^{*}$ & \\
\hline Moderadamente alto & $452(24,5)^{*}$ & $149(3,6)$ & \\
\hline Alto/excesso & $298(16,2)^{*}$ & $33(0,8)$ & \\
\hline Muito alto/obeso & $182(9,9)^{*}$ & $4(0,1)$ & \\
\hline Classificação da pressão arterial & & & $<0,001$ \\
\hline Ótima & $1(0,1)$ & $23(0,6)^{*}$ & \\
\hline Normal & $778(42,2)$ & $2.408(58,9)^{*}$ & \\
\hline Limítrofe & $409(22,2)$ & $867(21,2)$ & \\
\hline Hipertensão estágio I & $192(10,4)^{*}$ & $288(7,0)$ & \\
\hline Hipertensão estágio II & $465(25,2)^{*}$ & $505(12,3)$ & \\
\hline
\end{tabular}

\%GC percentual de gordura corporal. \# Teste qui-quadrado de Pearson. * Associação significativa pelo teste dos resíduos ajustados a 5\% de significância. 


\section{DISCUSSÃO}

Embora já existam estudos populacionais em diversas regiões do Rio Grande do Sul sobre o perfil nutricional de crianças e adolescentes, o presente estudo destaca-se por avaliar uma significante amostra, analisar maior número de variáveis antropométricas e investigar a associação dessas variáveis com a PA. Os resultados apresentados revelam dados alarmantes sobre obesidade, gordura corporal e obesidade central em crianças e adolescentes que vivem naquela região interiorana. Os dados condizem com a mudança de hábitos nutricionais vistos mundialmente.

Em um estudo realizado na cidade de São Leopoldo, RS, com 782 escolares do primeiro ano das escolas municipais de ensino fundamental, foi encontrada prevalência de excesso de peso de $38,1 \%$ [19], um pouco mais alta do que a encontrada no presente estudo. Em Santa Cruz do Sul, RS, Reuter et al. [20] encontraram em 658 estudantes de sete a 17 anos da zona urbana e rural uma prevalência de $29,5 \%$ de excesso de peso. Portanto, políticas públicas com o objetivo de diminuir a prevalência de obesidade infantil são importantes.

Um estudo realizado em escolas rurais de Santa Cruz do Sul, RS, avaliou obesidade e PA em 726 escolares (350 do sexo masculino e 376 do sexo feminino) e encontrou $14,5 \%$ com PAS elevada e $16,3 \%$ com PAD elevada, valores semelhantes aos encontrados neste estudo [21].

No presente estudo, quando somadas as classes de valores limítrofes e hipertensão estágios I e II, e estratificadas por sexo, encontrou-se prevalência maior se comparada com a encontrada no estudo de Burgos et al. [22], que avaliou 1.666 escolares com idades entre sete e 17 anos (873 do sexo masculino e 793 do sexo feminino). Esses autores classificaram a PA pelos percentis 90 e 95 para faixa limítrofe e hipertensão, e encontraram um percentual de escolares limítrofes e hipertensos de $5,2 \%$ e $8,7 \%$ para meninos e meninas, respectivamente. A PA foi também avaliada em 1.455 crianças e adolescentes (741 do sexo feminino e 714 do sexo masculino) de 10 a 17 anos de escolas públicas de Uruguaiana, RS, encontrando-se uma prevalência de PAS e de PAD elevadas de $16,4 \%$ para as meninas e de $18,5 \%$ para os meninos [23].

Sabe-se que alterações de pressão arterial potencializam o risco de complicações cardiovasculares na infância e na adolescência e, além disso, crianças/ adolescentes que apresentam alteração de PA tendem a manter esse quadro na vida adulta [24]. Os achados do presente estudo condizem com a literatura em relação às frequências de hipertensão arterial encontradas em crianças e em adolescentes no país, o que é preocupante. As associações entre níveis pressóricos aumentados e excesso de peso e de gordura abdominal são pontos importantes para o entendimento da causa da hipertensão em populações jovens. São múltiplas as causas de hipertensão nessa população, porém as mais comuns são o excesso de peso e o excesso de consumo alimentar de sódio [25]. Estes resultados confirmam a necessidade do uso de mais de uma variável antropométrica para distinguir precocemente riscos associados às doenças cardiovasculares.

Estudantes das escolas particulares apresentaram maior proporção de excesso de peso e crianças de escolas estaduais apresentaram maior proporção de magreza no presente estudo. Assim também, em um estudo que verificou a prevalência de sobrepeso e obesidade em escolares da rede pública e particular da cidade de Fortaleza, os autores encontraram maiores valores para as crianças de rede particular de ensino [26]. Segundo Miranda et al. [27], que buscaram diagnosticar e comparar a prevalência de sobrepeso e de obesidade em escolares da rede pública e privada, o índice de subnutrição foi menor em escolas privadas do que em escolas públicas. Além disto, na escola privada os autores acharam valores menores de crianças eutróficas em relação às crianças com excesso de peso.

No presente estudo verificou-se associação de hipertensão arterial com sobrepeso, obesidade e obesidade central. De acordo com Ribas et al. [28], existe associação entre excesso de peso e outros fatores de risco para síndrome metabólica, como exemplo, dislipidemia, hipertensão arterial, resistência à insulina e diabetes mellitus tipo 2. Um estudo realizado com crianças/adolescentes com idades entre 10 e 13 anos matriculados em instituições da rede pública de ensino da cidade de Londrina, PR, observou associação entre obesidade abdominal e ocorrência de hipertensão arterial [29]. Os resultados encontrados no presente estudo trazem uma grande preocupação, tendo em vista que crianças com obesidade central, inativos fisicamente e hipertensos tendem a se tornar adultos também obesos, inativos e hipertensos [30].

Um dos fatores limitantes do estudo foi não contemplar indicadores dietéticos, como o consumo de calorias, a qualidade lipídica, o consumo de carboidratos simples, o teor de sódio da dieta, o consumo de álcool, a ingestão de fibras e o consumo de probióticos, para o entendimento da alta prevalência de sobrepeso e obesidade. Além disto, outro fator limitante foi não contemplar a avaliação dos níveis 
de atividade física, uma vez que o comportamento sedentário ou inatividade física podem ter influência sobre o estado nutricional e a pressão arterial $[9,20]$.

Conclui-se que na população estudada de crianças e adolescentes, a hipertensão arterial associou-se com sobrepeso, obesidade e a obesidade central. Essa população necessita resoluções sobre condutas terapêuticas para minimizar danos a médio e longo prazo. Além disso, acredita-se que estudos longitudinais epidemiológicos com propostas preventivas, que visem o monitoramento do estado nutricional por meio de parâmetros antropométricos, geralmente com baixo custo, sejam fundamentais para minimizar a ocorrência de doenças crônicas não transmissíveis nos adultos. Estratégias públicas, como contratação de nutricionistas para atuarem mais diretamente na merenda escolar, criação de projetos de educação continuada para familiares, professores e merendeiras, podem ser estratégias efetivas para modificação do quadro vigente e promoção da saúde dessas e outras crianças e adolescentes.

\section{NOTAS}

\section{Apoio financeiro}

Este estudo não recebeu apoio financeiro de fontes externas.

\section{Declaração de conflito de interesses}

As autoras declaram não haver conflitos de interesses relevantes ao conteúdo deste estudo, informam ter tido acesso a todos os dados obtidos e assumem completa responsabilidade pela integridade dos resultados.

\section{REFERÊNCIAS}

1. World Health Organization. Global Strategy on Diet, Physical Activity and Health [Internet]. [cited 2017]. Available from: http://www.who.int/dietphysicalactivity/childhood what/en/

2. Wilmore JH, Costill DL, Kenney WL. Fisiologia do Esporte e do Exercício. 4aㅡ ed. Barueri: Manole; 2010.

3. Instituto de Pesquisa Econômica Aplicada. Objetivos de Desenvolvimento do Milênio: Relatório Nacional de Acompanhamento. Brasília: IPEA; 2010.

4. Vasconcelos AMN, Gomes MMF. Transição demográfica: a experiência brasileira. Epidemiol Serv Saúde. 2012;21(4):10. https://doi.org/10.5123/S1679-49742012000400003

5. Kac G, Velásquez-Meléndez G. A transição nutricional e a epidemiologia da obesidade na América Latina. Cad Saúde Pública, 2003;19(1):S4-S5. https://doi.org/10.1590/S0102-311X2003000700001

6. Rivera JA, de Cossío TG, Pedraza LS, Aburto TC, Sánchez TG, Martorell R. Childhood and adolescent overweight and obesity in Latin America: a systematic review Lancet Diabetes Endocrinol. 2014 Apr;2(4):321-32. https://doi.org/10.1016/ S2213-8587(13)70173-6

7. Rosaneli CF, Auler F, Manfrinato CB, Rosaneli CF, Sganzerla C, Bonatto MG, Cerqueira MLW, Oliveira AAB, OliveiraNetto ER, Faria-Neto JR. Avaliação da prevalência e de determinantes nutricionais e sociais do excesso de peso em uma população de escolares: análise transversal em 5.037 crianças. Rev Assoc Med Bras 2012;58(4):472-6. https://doi. org/10.1590/S0104-42302012000400019

8. Leal VS, Lira PIC, Oliveira JS, Menezes RCE, Sequeira LAS, Arruda Neto MA, Andrade SLLS, Batista Filho M. Excesso de peso em crianças e adolescentes no Estado de Pernambuco, Brasil: prevalência e determinantes. Cad Saúde Pública. 2012;28(6):1175-82. https://doi.org/10.1590/S0102-311X2012000600016

9. Reuter CP, Burgos LT, Camargo MD, Possuelo LG, Reckziegel MB, Reuter ÉM, Meinhardt FP, Burgos MS. Prevalence of obesity and cardiovascular risk among children and adolescents in the municipality of Santa Cruz do Sul, Rio Grande do Sul. Sao Paulo Med J. 2013;131(5):323-30. https://doi.org/10.1590/1516-3180.2013.1315518

10. World Health Organization. Global Status Report on noncommunicable diseases [Internet]. 2014 [cited 2017 Feb 9]. Available from: http://www.who.int/nmh/publications/ncd-status-report-2014/en/

11. Ministério da Saúde (BR). Secretaria de Atenção à Saúde. Departamento de Atenção Básica. Saúde da criança: crescimento e desenvolvimento [Internet]. Brasília: Ministério da Saúde; 2012 [cited 2017]. (Cadernos de Atenção Básica, 33). Available from: http://189.28.128.100/dab/docs/publicacoes/cadernos_ab/caderno_33.pdf

12. World Health Organization. WHO Child Growth Standards: length/height-for-age, weight-for age, weight-for-length, weight-for-height and body mass index-for-age. Methods and development. Geneva: WHO; 2006.

13. McCarthy HD, Jarret KV, Crawley HF. The development of waist circumference percentiles in British children aged 5.0-16.9 y. Eur J Clin Nutr. 2001;55(10):902-7. https://doi.org/10.1038/sj.ejen.1601240

14. Alberti KG, Eckel RH, Grundy SM, Zimmet PZ, Cleeman JI, Donato KA, Fruchart JC, James WP, Loria CM, Smith SC Jr; International Diabetes Federation Task Force on Epidemiology and Prevention; Hational Heart, Lung, and Blood Institute; American Heart Association; World Heart Federation; International Atherosclerosis Society; International Association for the Study of Obesity. Harmonizing the metabolic syndrome: A joint interim statement of the international diabetes federation task force on epidemiology and prevention; national heart, lung, and blood institute; American heart association; world heart federation; international atherosclerosis society; and international association for the study of obesity. Circulation. 2009 Oct 20;120(16):1640-5 https://doi.org/10.1161/CIRCULATIONAHA.109.192644 
15. Slaughter MH, Lohman TG, Boileau RA, Horswill CA, Stillman RJ, Van Loan MD, Bemben DA. Skinfold equations for estimation of body fatness in children and youth. Hum Biol. 1988 Oct;60(5):709-23.

16. Lohman TG. The use of skinfold to estimate body fatness on children and youth J Physical Educ Recreation Dance 1987;58:98-102. https://doi.org/10.1080/07303084.1987.10604383

17. VI Diretrizes Brasileira de Hipertensão. Arq Bras Cardiol. 2010;95(supl 1):1-51.

18. National High Blood Pressure Education Program Working Group on High Blood Pressure in Children and Adolescents. The fourth report on the diagnosis, evaluation, and treatment of high blood pressure in children and adolescents. Pediatrics. 2004;114:555-76. https://doi.org/10.1542/peds.114.2.S2.555

19. Vicenzi K, Henn RL, Weber AP, Backes V, Paniz VMV, Donatti T, Olinto MTA. Insegurança alimentar e excesso de peso em escolares do primeiro ano do Ensino Fundamental da rede municipal de São Leopoldo, Rio Grande do Sul, Brasil. Cad. Saúde Pública. 2015;31(5):1084-94 https://doi.org/10.1590/0102-311X00055914

20. Reuter CP, Burgos MS, Pritsch CVM, Silva PT, Marques KC, Souza S, Pasa L, Silva R, Muradás R. Obesidade, aptidão cardiorrespiratória, atividade física e tempo de tela em escolares da zona urbana e rural de Santa Cruz do Sul-RS. Cinergis. 2015;16(1):52-6. https://doi.org/10.17058/cinergis.v16i1.6271

21. Hoehr CF, Reuter CP, Tornquist L, Nunes HMB, Burgos MS. Prevalência de obesidade e hipertensão arterial em escolares: estudo comparativo entre escolas rurais do município de Santa Cruz do Sul/RS. Rev Epidemiol Control Infect. 2014;4(2):122-6. https://doi.org/10.17058/reci.v4i2.4772

22. Burgos MS, Reuter CP, Burgos LT, Pohl HH, Pauli LT, Horta JA, Reckziegel MB, Franke SI, Prá D, Camargo M. Uma análise entre índices pressóricos, obesidade e capacidade cardiorrespiratória em escolares. Arq Bras Cardiol. 2010;94(6):788-93. https://doi.org/10.1590/S0066-782X2010005000046

23. Bergmann MLA, Graup S, Bergmann GG. Pressão arterial elevada em adolescentes e fatores associados: um estudo de base escolar em Uruguaiana, Rio Grande do Sul, 2011. Rev Bras Saude Mater Infant. 2015;15(4):377-87. https://doi. org/10.1590/S1519-38292015000400002

24. World Health Organization. Cardiovascular disease. A global brief on hypertension [Internet]: silent killer, global public health crisis. Geneva: WHO; 2013 [cited 2017]. Available from: http://www.who.int/cardiovascular_diseases/publications/ global_brief_hypertension/en

25. Rao G. Diagnosis, Epidemiology, and Management of Hypertension in Children. Pediatrics. 2016 Aug;138(2). pii: e20153616. https://doi.org/10.1542/peds.2015-3616

26. Paula FAR, Lamboglia CMGF, Silva VTBL, Monteiro MS, Moreira AP, Pinheiro MHNP, Silva CAB. Prevalência de sobrepeso e obesidade em escolares da rede pública e particular da cidade de Fortaleza. Rev Bras Promoc Saúde. 2015;27(4):455-61. https://doi.org/10.5020/18061230.2014.p455

27. Miranda JMQ, Palmeira MV, Polito LFT, Brandão MRF, Bocalini DS, Figueira J. Ponciano K, Vichi RB. Prevalência de sobrepeso e obesidade infantil em instituições de ensino: públicas vs. privadas. Rev Bras Med Esporte. 2015;21(2):104-7. https://doi.org/10.1590/1517-869220152102143660

28. Ribas SA, Santana da Silva LC. Anthropometric indices: predictors of dyslipidemia in children and adolescents from north of Brazil. Nutr Hosp. 2012 July-Aug;27(4):1228-35.

29. Casonatto J, Ohara D, Christofaro DGD, Fernandes RA, Milanez V, Dias DF, Freitas Júnior I, Oliveira AR. Pressão arterial elevada e obesidade abdominal em adolescentes. Rev Paul Pediatr. 2011;29(4):567-71. https://doi.org/10.1590/ S0103-05822011000400015

30. Siqueira DGB, Souza RKT, Mesas AE, Santos HG, Bortoletto MSS. Diferenças entre sexos nos determinantes da obesidade abdominal em adultos de 40 anos ou mais: estudo de base. Rev Nutr. 2015;28(5):485-96. https://doi.org/10.1590/141552732015000500003 